\title{
Asymptotic Solutions of Numerical Transport Problems in Optically Thick, Diffusive Regimes II
}

\author{
EDWARD W. LARSEN \\ Department of Nuclear Engineering, The University of Michigan, \\ Ann Arbor, Michigan 48109
}

AND

J. E. MOREL

University of California, Los Alamos National Laboratory, Radiation Transport Group, X-6, Los Alamos, New Mexico 87545

Received December 11, 1987; revised August 11, 1988

\begin{abstract}
In a recent article (Larsen, Morel, and Miller, $J$.Comput. Phys. 69, 283 (1987)), a theoretical method is described for assessing the accuracy of transport differencing schemes in highly scattering media with optically thick spatial meshes. In the present article, this method is extended to enable one to determine the accuracy of such schemes in the presence of numerically unresolved boundary layers. Numerical results are presented that demonstrate the validity and accuracy of our analysis. (1) 1989 Academic Press, Inc.
\end{abstract}

\section{INTRODUCTION}

In the last four decades, much effort has been spent on the development and analysis of numerical methods for discrete ordinates problems. The motivation for this work has come largely from the nuclear power industry, for which accurate solutions of geometrically complex neutron transport problems are required. Much of this work is described in the recent book by Lewis and Miller [2]. With few exceptions, the spatial differencing schemes that have been widely used produce solutions whose errors tend to zero when the optical thickness of the spatial cells $\tau=\sigma h$ tends to zero. Theoretical studies of these differencing schemes have focused on optically thin cells, and error estimates of the form $O\left(\tau^{n}\right)$ for $\tau \ll 1$, obtained by the estimation of truncation errors, are typical results of these studies. A representative sampling of this work is given in [3-6]. The optically thin regime is relevant for neutron transport, where one can usually afford the computer storage necessary to achieve spatial meshes satisfying $\tau<1$.

In recent years, however, neutronics methods have been applied to electron and thermal radiation transport problems, which are optically much thicker than 
neutron transport problems. Here computer storage limitations frequently require the use of spatial meshes which are extremely thick by neutronics standards; typical values of $\tau$ for electron transport [7] are $\tau=O\left(10^{2}\right)$, and for thermal radiative transfer [8] are $\tau=O\left(10^{6}\right)$. One may expect accuracy in such problems with optically thick cells because away from boundary layers, the scale length over which the solutions vary by an $O(1)$ amount constitutes many mean free paths. In the case of electron transport, this scale length is associated with the transport-corrected mean free path, and in the case of photon transport, with the diffusion length. Nevertheless, it is clear that theoretical results for $\tau \ll 1$ cannot be relevant in assessing the accuracy of transport differencing schemes for such optically thick meshes.

In a recent article [1], a $\tau \gg 1$ analysis for "diffusive" problems is developed, and in this article we extend this analysis to problems containing boundary layers which are not resolved by the spatial mesh. The traditional $\tau \ll 1$ analyses are based on the estimation of truncation errors; the $\tau \gg 1$ analysis in [1] and this article is instead based on an asymptotic expansion. This expansion was first applied $[9,10]$ to the following analytic transport problem. In this article, we will consider discretized versions of this problem:

$$
\begin{aligned}
\mu \frac{\partial}{\partial x} \psi(x, \mu)+\frac{\sigma_{T}(x)}{\varepsilon} \psi(x, \mu) & \\
=\left[\frac{\sigma_{T}(x)}{\varepsilon}-\varepsilon \sigma_{A}(x)\right] \frac{1}{2} \int_{-1}^{1} \psi\left(x, \mu^{\prime}\right) d \mu^{\prime}+\frac{\varepsilon Q(x)}{2}, \quad 0<x<L, & \\
\psi(0, \mu) & =f(\mu), \quad 0<\mu \leqslant 1, \\
\psi(L, \mu) & =g(\mu), \quad-1 \leqslant \mu<0 .
\end{aligned}
$$

This problem is written in standard neutronics notation, except for the scaling in the small dimensionless parameter $\varepsilon$, which is fully discussed in [1]. Thus, $\psi(x, \mu)$ is the angular flux at the point $x$ in the "direction" $\mu, \sigma_{T}(x)$ is the scaled total cross section, $\sigma_{A}(x)$ is the scaled absorption cross section, $Q(x)$ is a scaled source, and $\phi(x)$ is the scalar flux. These quantities are required to be $O(1)$ as $\varepsilon \rightarrow 0$. Thus, Eq. (1.1) is scaled in such a way that the physical total cross section $\varepsilon^{-1} \sigma_{T}$ is large, while the physical absorption cross section $\varepsilon \sigma_{A}$ and the physical source $\varepsilon Q$ are small and comparable in size. (We refer the reader to [1] for a thorough discussion, which we cannot reproduce here, on the rationale for the scaling in Eq. (1.1a), and for a more detailed presentation of the ideas underlying our asymptotic methodology.) We shall assume, for simplicity, that $\sigma_{T}(x), \sigma_{A}(x)$, and $Q(x)$ are continuous functions of $x$, so boundary layers in the above problem can occur only at the cell edges $x=0$ and $x=L$.

As $\varepsilon \rightarrow 0$, the solution of problem $(1.1)$ has been shown $[9,10]$ to satisfy:

$$
\psi(x, \mu)=\frac{1}{2} \phi(x)+O(\varepsilon), \quad 0<x_{0} \leqslant x \leqslant x_{1}<L,
$$


where $\phi(x)$ satisfies the diffusion problem

$$
\begin{gathered}
-\frac{d}{d x} \frac{1}{3 \sigma_{T}(x)} \frac{d}{d x} \phi(x)+\sigma_{A}(x) \phi(x)=Q(x), \\
\frac{1}{2} \phi(0)=\int_{0}^{1} W(\mu) f(\mu) d \mu \\
\frac{1}{2} \phi(L)=\int_{-1}^{0} W(-\mu) g(\mu) d \mu .
\end{gathered}
$$

In Eq. (1.2), $x_{0}$ and $x_{1}$ are arbitrary fixed points within the interval $[0, L]$; the result (1.2) is generally not valid arbitrarily close to $x=0$ and $x=L$, due to boundary layers of width $O(\varepsilon)$ which may exist in the transport solution, but which do not exist in the simpler diffusion solution. Also, in Eqs. (1.3b) and (1.3c),

$$
W(\mu)=\frac{\mu}{X(-\mu)}\left[\int_{0}^{1} \frac{s}{X(-s)} d s\right]^{-1},
$$

where $X(-\mu)$ is tabulated for $0 \leqslant \mu \leqslant 1$ in $[11$, Table L.14, p. 337]. (The title of this table incorrectly states that $X(\mu)$ is tabulated.) The function $W(\mu)$ is smooth and well approximated by the polynomial

$$
\tilde{W}(\mu)=0.956 \mu+1.565 \mu^{2},
$$

where

$$
\sup _{0 \leqslant \mu \leqslant 1}|W(\mu)-\tilde{W}(\mu)|=0.0035
$$

Equation (1.3a) is the standard diffusion approximation to the transport equation. The boundary conditions $(1.3 \mathrm{~b})$ and $(1.3 \mathrm{c})$ can be understood heuristically as follows. As $\varepsilon \rightarrow 0$, the incident flux $f(\mu)$ at $\mu=0$ "sees" a slab of infinitely large optical thickness, with infinitesimally small absorption and sources. In other words, it sees, in standard neutronics terminology, a " $\mathrm{c}=1$ " half space. (In our notation, the "scattering ratio" $c$ is given by $c=1-\varepsilon^{2} \sigma_{A} / \sigma_{T}$, and is asymptotically close to unity.) Thus, a few mean free paths to the right of $x=0$, the angular flux behaves as the solution of the $c=1$ half-space problem, i.e., as the isotropic constant given by the right side of Eq. (1.3b). Similarly, the incident flux $g(\mu)$ at $x=L$ "sees" the $c=1$ half space $x<L$, so the angular flux, a few mean free paths to the left of $x=L$, tends to the isotropic constant given by the right side of Eq. (1.3c).

In [1], the asymptotic expansion which leads from (1.1a) to (1.2) and (1.3a) is applied to the discretized version of the transport equation (1.1a) on a fixed spatial mesh. (In this earlier work, the boundary conditions (1.1b) and (1.1c) are not treated.) We note that as $\varepsilon \rightarrow 0$, each spatial cell becomes infinitely many mean free paths thick. If the asymptotic solution of this discretized equation converges as 
$\varepsilon \rightarrow 0$ to the solution of a stable and consistently discretized version of the diffusion equation (1.3a), then the transport differencing scheme is said in [1] to possess the "thick" diffusion limit, and the use of this transport differencing scheme on optically thick meshes is partially justified. The numerical result in [1], however, show that schemes possessing this thick diffusion limit do not always produce accurate solutions in thick diffusive problems. The extra condition which a differencing scheme must satisfy to guarantee an accurate numerical transport solution is for it to resolve, with a suitably fine spatial mesh, the transport boundary layers at exterior boundaries, where anisotropic incident fluxes may enter the system, and at interior boundaries between media with different material cross sections. In practice, computer storage limitations frequently do not allow the use of an optically thin spatial mesh, both throughout a system and in the vicinity of boundary layers. This is particularly true in multidimensional geometries. Therefore, the analysis in [1] does not fully apply to the "coarse mesh" discretizations frequently used for electron and photon transport problems because it does not assess the accuracy of a transport differencing scheme on a spatial mesh containing a numerically unresolved boundary layer. In this article, we extend the method in [1] to this type of problem.

To be specific, we consider the asymptotic expansion applied to the full problem (1.1), with the boundary conditions. We apply this expansion to the full discretized problem with a fixed spatial mesh, obtaining as $\varepsilon \rightarrow 0$ a set of equations for the unknowns in the discretization scheme. Again, in this limit, each spatial cell in the problem becomes infinitely many mean free paths thick. Away from $x=0$ and $x=L$, these equations either are or are not a stable and consistent discretization of the diffusion equation (1.3a); if they are, then the first necessary condition for the differencing scheme to be accurate for thick cells is met. For the cells adjoining $x=0$ and $x=L$, these equations take the form of boundary conditions which either do or do not closely approximate Eqs. (1.3b) and (1.3c); if they do, then the second necessary condition for the differencing scheme to be accurate for thick cells is met. The third and final condition for accuracy is that the transport spatial mesh be sufficiently fine that the discretized diffusion problem (1.3), resulting from the asymptotic expansion of the discretized transport problem (1.1), is adequately resolved on this mesh.

In this article we discuss in detail the diamond difference (DD) and linear discontinuous (LD) spatial differencing schemes $[2,12,13]$ for the standard discrete ordinates equations. (We also consider simple generalizations of the LD method and include a brief discussion of a "new" method described in [1].) Our conclusions are as follows:

(1) Although the cell-average DD fluxes possess the thick diffusion limit, the cell-edge fluxes do not (this was shown in [1]), and both can be quite inaccurate if either of the incident boundary fluxes is anisotropic.

(2) Although the "new" method in [1] has cell-edge and cell-average fluxes which possess the thick diffusion limit (this was derived in [1]), the boundary 
conditions for this diffusion equation become unbounded as $\varepsilon \rightarrow 0$ if either of the boundary fluxes is anisotropic. This explains the disastrous performance of this method in problem 4 of [1].

(3) The LD methods have cell-edge and cell-average fluxes which possess the thick diffusion limit, and these methods are surprisingly accurate for problems with anisotropic boundary fluxes.

(4) Among the differencing schemes considered here and in [1], the LD methods are the most accurate for optically thick cells with unresolved boundary layers. In fact, these methods are sufficiently accurate that they should be adequate for many practical applications.

A summary of the remainder of this article follows. In Section II we analyze the DD method; this was incompletely done in [1]. In Section III we summarized our results for the "new" method described in [1], and in Section IV we analyze the LD method. In Section $\mathrm{V}$ we consider numerical accuracy on the system boundaries, and in Section VI we present numerical calculations that demonstrate the validity of our theory. We conclude with a discussion in Section VII.

\section{The Diamond Difference Method}

The DD method is partially treated in [1]. To fully analyze this method, we consider $[1,2]$ the discrete ordinates, diamond-differenced form of problem $(1,1)$ :

$$
\begin{gathered}
\frac{\mu_{m}}{h_{j}}\left(\psi_{m, j+1 / 2}-\psi_{m, j-1 / 2}\right)+\frac{\sigma_{T j}}{\varepsilon} \psi_{m j}=\left(\frac{\sigma_{T j}}{\varepsilon}-\varepsilon \sigma_{A j}\right) \frac{1}{2} \sum_{n=1}^{N} \psi_{n j} w_{n}+\frac{\varepsilon Q_{j}}{2}, \\
\psi_{m j}=\frac{1}{2}\left(\psi_{m, j+1 / 2}+\psi_{m, j-1 / 2}\right), \\
\psi_{m, 1 / 2}=f_{m}, \quad \mu_{m}>0, \\
\psi_{m, J+1 / 2}=g_{m}, \quad \mu_{m}<0 .
\end{gathered}
$$

In these equations, we use any standard symmetric quadrature set $\left\{\mu_{m}, w_{m}\right\}$ of even order $N$, satisfying

$$
\sum_{n=1}^{N}\left(\mu_{n}\right)^{k} w_{n}= \begin{cases}2, & k=0, \\ 0, & k=1, \\ \frac{2}{3}, & k=2 .\end{cases}
$$

We choose a spatial mesh such that the $j$ th cell, for $1 \leqslant j \leqslant J$, is defined by $x_{j} \quad 1 / 2<x<x_{j+1 / 2}$ with $h_{j}=x_{j+1 / 2}-x_{j-1 / 2}=$ cell width, $x_{j}=\left(x_{j+1 / 2}+x_{j-1 / 2}\right) / 2=$ cell center, $x_{1 / 2}=0$ and $x_{J+1 / 2}=L$. Also, integer $\pm \frac{1}{2}$ subscripts refer to cell-edge quantities, and integer subscripts refer to cell-average quantities. 
To perform the asymptotic analysis, we introduce the ansatz

$$
\psi_{m} \approx \sum_{k=0}^{\infty} \varepsilon^{k} \psi_{m}^{(k)}
$$

for both the cell-edge fluxes $\psi_{m, j+1 / 2}$ and cell-average fluxes $\psi_{m j}$ into Eqs. (2.1), equate the coefficients of $\varepsilon^{-1}, \varepsilon^{0}$, and $\varepsilon^{1}$, and then solve the resulting system of equations.

The sole $O\left(\varepsilon^{-1}\right)$ equation is

$$
\sigma_{T_{j}}\left(\psi_{m j}^{(0)}-\frac{1}{2} \sum_{n=1}^{N} \psi_{n j}^{(0)} w_{n}\right)=0
$$

which has the isotropic solution

$$
\psi_{m j}^{(0)}=\frac{1}{2} \phi_{j}^{(0)},
$$

where $\phi_{j}^{(0)}$ is, at this point, undetermined.

The four $O\left(\varepsilon^{0}\right)$ equations are

$$
\begin{gathered}
\frac{1}{2} \phi_{j}^{(0)}=\frac{1}{2}\left(\psi_{m, j+1 / 2}^{(0)}+\psi_{m, j-1 / 2}^{(0)}\right), \\
\sigma_{T_{J}}\left(\psi_{m j}^{(1)}-\frac{1}{2} \sum_{n=1}^{N} \psi_{n j}^{(1)} w_{n}\right)=-\frac{\mu_{m}}{h_{j}}\left(\psi_{m, j+1 / 2}^{(0)}-\psi_{m, j-1 / 2}^{(0)}\right), \\
\psi_{m, 1 / 2}^{(0)}=f_{m}, \quad \mu_{m}>0, \\
\psi_{m, J+1 / 2}^{(0)}=g_{m}, \quad \mu_{m}<0,
\end{gathered}
$$

which have been written in the order in which they will be solved. To treat the first of these equations, let us make the following definitions:

$$
\begin{aligned}
\phi_{j+1 / 2}^{(0)} & \equiv \sum_{n=1}^{N} 3 \mu_{n}^{2} \psi_{n, j+1 / 2}^{(0)} w_{n}, \\
\eta_{m, j+1 / 2}^{(0)} & \equiv 2 \psi_{m, j+1 / 2}^{(0)}-\phi_{j+1 / 2}^{(0)} .
\end{aligned}
$$

The first of these is somewhat curious, but it will be justified in what follows. These two definitions imply that

$$
\sum_{m=1}^{N} \mu_{m}^{2} \eta_{m, j+1 / 2}^{(0)} w_{m}=0
$$

and that Eq. (2.6a) can be written

$$
\phi_{j}^{(0)}=\frac{1}{2}\left(\phi_{j+1 / 2}^{(0)}+\phi_{j-1 / 2}^{(0)}\right)+\frac{1}{2}\left(\eta_{m, j+1 / 2}^{(0)}+\eta_{m, j-1 / 2}^{(0)}\right) .
$$


Multiplying this last equation by $\mu_{m}^{2} w_{m}$, summing over $m$, and using Eq. (2.9), we obtain

$$
\phi_{j}^{(0)}=\frac{1}{2}\left(\phi_{j+1 / 2}^{(0)}+\phi_{j-1 / 2}^{(0)}\right)
$$

and thus

$$
0=\eta_{m, j+1 / 2}^{(0)}+\eta_{m, j-1 / 2}^{(0)}
$$

implying

$$
\eta_{m, j+1 / 2}^{(0)}=(-1)^{j} \eta_{m}^{(0)}
$$

Thus, Eqs. (2.8) and (2.9) can be rewritten as

$$
\begin{aligned}
\psi_{m, j+1 / 2}^{(0)} & =\frac{1}{2}\left[\phi_{j+1 / 2}^{(0)}+(-1)^{j} \eta_{m}^{(0)}\right], \\
0 & =\sum_{m=1}^{N} \mu_{m}^{2} \eta_{m}^{(0)} w_{m} .
\end{aligned}
$$

Now, introducing Eq. (2.14) into (2.6b), we get

$$
\sigma_{T j}\left(\psi_{m j}^{(1)}-\frac{1}{2} \sum_{n=1}^{N} \psi_{n j}^{(1)} w_{n}\right)=-\frac{\mu_{m}}{2 h_{j}}\left[\phi_{j+1 / 2}^{(0)}-\phi_{j-1 / 2}^{(0)}+2(-1)^{j} \eta_{m}^{(0)}\right] .
$$

Multiplying this equation by $w_{m}$ and summing over $m$, the left side vanishes, and we obtain the following solvability condition, which must hold for a solution of Eq. (2.16) to exist:

$$
0=\sum_{m=1}^{N} \mu_{m} \eta_{m}^{(0)} w_{m}
$$

Requiring this condition to hold, we find the general solution of Eq. (2.16) to be

$$
\psi_{m j}^{(1)}=\frac{1}{2} \phi_{j}^{(1)}-\frac{\mu_{m}}{2 \sigma_{7 j} h_{j}}\left(\phi_{j+1 / 2}^{(0)}-\phi_{j-1 / 2}^{(0)}+2(-1)^{j} \eta_{m}^{(0)}\right),
$$

where $\phi_{j}^{(1)}$ is, at this point, undetermined.

Next, we introduce Eq. (2.14) into Eqs. (2.6c) and (2.6d), yielding

$$
\begin{array}{rlrl}
\phi_{1 / 2}^{(0)}+\eta_{m}^{(0)} & =2 f_{m}, & & \mu_{m}>0, \\
\phi_{J+1 / 2}^{(0)}+(-1)^{J} \eta_{m}^{(0)}=2 g_{m}, & & \mu_{m}<0 .
\end{array}
$$

These two equations imply:

$$
\eta_{m}^{(0)}= \begin{cases}2 f_{m}-\phi_{1 / 2}^{(0)}, & \mu_{m}>0, \\ (-1)^{J}\left(2 g_{m}-\phi_{J+1 / 2}^{(0)}\right), & \mu_{m}<0 .\end{cases}
$$


Introducing this result into Eqs. (2.15) and (2.17), we obtain two equations for the two unknowns $\phi_{1 / 2}^{(0)}$ and $\phi_{J+1 / 2}^{(0)}$. Solving for these unknowns, we obtain

$$
\begin{aligned}
\frac{1}{2} \phi_{1 / 2}^{(0)}= & \sum_{\mu_{m}>0}\left(\frac{\mu_{m}}{\gamma}+\frac{3}{2} \mu_{m}^{2}\right) f_{m} w_{m} \\
& +(-1)^{J} \sum_{\mu_{m}<0}\left(-\frac{\left|\mu_{m}\right|}{\gamma}+\frac{3}{2} \mu_{m}^{2}\right) g_{m} w_{m}, \\
\frac{1}{2} \phi_{J+1 / 2}^{(0)}= & \sum_{\mu_{m}<0}\left(\frac{\left|\mu_{m}\right|}{\gamma}+\frac{3}{2} \mu_{m}^{2}\right) g_{m} w_{m} \\
& +(-1)^{J} \sum_{\mu_{m}>0}\left(-\frac{\mu_{m}}{\gamma}+\frac{3}{2} \mu_{m}^{2}\right) f_{m} w_{m},
\end{aligned}
$$

where the constant $\gamma$ is defined by

$$
\gamma=2 \sum_{\mu_{m}>0} \mu_{m} w_{m} \approx 1
$$

To proceed, we must consider the $O\left(\varepsilon^{1}\right)$ equations. However, we only need the $O\left(\varepsilon^{1}\right)$ part of Eqs. (2.1a) and (2.1b):

$$
\begin{aligned}
\sigma_{T j}\left(\psi_{m i}^{(2)}-\frac{1}{2} \sum_{n=1}^{N} \psi_{n j}^{(2)} w_{n}\right)= & -\frac{\mu_{m}}{h_{j}}\left(\psi_{m . j+1 / 2}^{(1)}-\psi_{m, j-1 / 2}^{(1)}\right) \\
& +\frac{1}{2}\left(-\sigma_{A j} \phi_{j}^{(0)}+Q_{j}\right), \\
\psi_{m j}^{(1)}= & \frac{1}{2}\left(\psi_{m, j+1 / 2}^{(1)}+\psi_{m, j-1 / 2}^{(1)}\right) .
\end{aligned}
$$

Multiplying Eq. (2.23) by $w_{m}$ and summing over $m$, the left side vanishes, and we obtain

$$
\vartheta_{j+1 / 2}^{(1)}-\vartheta_{j-1 / 2}^{(1)}=h_{j}\left(-\sigma_{A j} \phi_{j}^{(0)}+Q_{j}\right)
$$

where

$$
\vartheta_{j+1 / 2}^{(1)} \equiv \sum_{m=1}^{N} \mu_{m} \psi_{m, j+1 / 2}^{(1)} w_{m}
$$

Summing Eq. (2.25) over the $j$ th and $(j+1)$ th cells, we obtain

$$
\begin{aligned}
\vartheta_{j+3 / 2}^{(1)}-\vartheta_{j-1 / 2}^{(1)}= & -\left(\sigma_{A, j+1} h_{j+1} \phi_{j+1}^{(0)}+\sigma_{A, j} h_{j} \phi_{j}^{(0)}\right) \\
& +\left(h_{j+1} Q_{j+1}+h_{j} Q_{j}\right) .
\end{aligned}
$$


Similarly, Eqs. (2.24), (2.18), and (2.9) imply

$$
\begin{aligned}
\vartheta_{j+3 / 2}^{(1)}-\vartheta_{j-1 / 2}^{(1)}= & 2 \sum_{m=1}^{N} \mu_{m}\left(\psi_{m, j+1}^{(1)}-\psi_{m, j}^{(1)}\right) w_{m} \\
= & -\frac{2}{3 \sigma_{T, j+1} h_{j+1}}\left(\phi_{j+3 / 2}^{(0)}-\phi_{j+1 / 2}^{(0)}\right) \\
& +\frac{2}{3 \sigma_{T, j} h_{j}}\left(\phi_{j+1 / 2}^{(0)}-\phi_{j-1 / 2}^{(0)}\right) .
\end{aligned}
$$

These two equations hold for any "interior" value of $j, 2 \leqslant j \leqslant J-1$. Equating their right sides, we obtain

$$
\begin{aligned}
& -\frac{1}{3 \sigma_{T, j+1} h_{j+1}}\left(\phi_{j+3 / 2}^{(0)}-\phi_{j+1 / 2}^{(0)}\right)+\frac{1}{3 \sigma_{T, j} h_{j}}\left(\phi_{j+1 / 2}^{(0)}-\phi_{j-1 / 2}^{(0)}\right) \\
& +\frac{1}{4}\left[\sigma_{A, j+1} h_{j+1}\left(\phi_{j+3 / 2}^{(0)}+\phi_{j+1 / 2}^{(0)}\right)+\sigma_{A, j} h_{j}\left(\phi_{j+1 / 2}^{(0)}+\phi_{j-1 / 2}^{(0)}\right)\right] \\
& =\frac{1}{2}\left(h_{j+1} Q_{j+1}+h_{j} Q_{j}\right), \quad 2 \leqslant j \leqslant J-1 .
\end{aligned}
$$

We can now summarize our results. To leading order, the cell-average angular fluxes are given by Eqs. (2.5) and (2.11):

$$
\psi_{m j}=\frac{1}{4}\left(\phi_{j+1 / 2}^{(0)}+\phi_{j-1 / 2}^{(0)}\right)+O(\varepsilon)
$$

and the cell-edge fluxes by Eq. (2.14),

$$
\psi_{m, j+1 / 2}=\frac{1}{2}\left[\phi_{j+1 / 2}^{(0)}+(-1)^{j} \eta_{m}^{(0)}\right]+O(\varepsilon),
$$

where $\phi_{j+1 / 2}^{(0)}$ are determined by Eqs. (2.21) and (2.29), and $\eta_{m}^{(0)}$ by Eq. (2.20).

It is clear that Eq. (2.29) is a stable and consistently discretized version of the diffusion equation (1.3a). Therefore, the cell-average fluxes have the thick diffusion limit, whereas the cell-edge fluxes generally do not, unless $\eta_{m}^{(0)}=0$.

If we now ask whether the boundary conditions $(2.21)$ closely approximate Eqs. (1.3), the answer is that they do not, unless (see Eq. (1.5)) $f_{m}$ and $g_{m}$ happen to satisfy

$$
0=\sum_{\mu_{m}>0}\left(-\frac{\mu_{m}}{\gamma}+\frac{3}{2} \mu_{m}^{2}\right) f_{m} w_{m}=\sum_{\mu_{m}<0}\left(-\frac{\left|\mu_{m}\right|}{\gamma}+\frac{3}{2} \mu_{m}^{2}\right) g_{m} w_{m} .
$$

These conditions hold if $f_{m}$ and $g_{m}$ are isotropic, but they do not hold in general. Also, we note that for arbitrary problems, the boundary conditions (2.21) depend on whether there is an even or an odd number $J$ of spatial cells in the system! Moreover, the variation on the left boundary (for odd or even $J$ ) is due solely to 
an anisotropic incident boundary flux on the right boundary, and the variation (for odd or even $J$ ) on the right boundary is due solely to an anisotropic incident boundary flux on the left boundary. Equally interesting is that fact that if the $(-1)^{J}$ terms in Eqs. (2.21) were not present, then by Eqs. (1.3) and (1.5), we would have an excellent representation of the correct boundary conditions (1.3). However, this is a moot point because these $(-1)^{J}$ terms in fact are present.

The definition (2.7) seems, at first glance, to be a bit arbitrary; the more obvious choice would be

$$
\phi_{j+1 / 2}^{(0)}=\sum_{n=1}^{N} \psi_{n, j+1 / 2}^{(0)} w_{n} .
$$

One can in fact implement our analysis with this (or any other) definition of $\phi_{j+1 / 2}^{(0)}$, but the resulting algebraic details actually become considerably more complicated, and the results, in the end, are identical with those derived above. We have opted in this article to use the non-obvious definition (2.7) because it leads to the simplest analysis.

In conclusion, the DD cell-average fluxes are determined by a discretized version of the correct diffusion equation. However, the boundary conditions for this equation are generally incorrect if the boundary fluxes are anisotropic. Moreover, these incorrect boundary conditions vary, depending on whether the system has an odd or an even number of cells.

\section{THE "New" MeTHOD}

In [1], a "new" differencing scheme is discussed for which, unlike the DD method, the cell-edge and cell-average fluxes both have the optically thick diffusion limit. However, in problem 4 of [1], consisting of a normally incident flux on a slab with optically thick spatial cells, this method yields a solution with an extremely large (and inaccurate) amplitude. We have applied our full asymptotic analysis to this problem. The details of this analysis will not be described here, but the main results are as follows. If either of the incident boundary fluxes is anisotropic, then the ansatz (2.3) for the cell-average and cell-edge fluxes does not lead to a consistent asymptotic solution, whereas the more general ansatz

$$
\psi_{m} \approx \sum_{k=-1}^{\infty} \varepsilon^{k} \psi_{m}^{(k)}
$$

does. The difference between Eqs. (2.3) and (3.1) is that in Eq. (3.1), $\psi_{m} \rightarrow \infty$ as $\varepsilon \rightarrow 0$, and indeed, this is consistent with the numerical results reported in problem 4 of [1].

This analysis shows that although the cell-edge and cell-average fluxes generated by the "new" scheme do satisfy the correct diffusion equation in the optically thick, 
diffusive limit, the scheme interacts with unresolved transport boundary layers so poorly that the boundary conditions for the diffusion solution are extremely inaccurate. Clearly, the "new" scheme is not suitable for general, optically thick problems. (Modifications in this scheme, however, have been devised which correct some of these deficiencies $[14,15]$.)

The results in this and the previous section emphasize the main theme of this article: to obtain accurate transport solutions on optically thick and diffusive cells, a differencing scheme must have the optically thick diffusion limit and the boundary conditions to go with this diffusion equation must be accurate. In the next section, we consider LD schemes, for which both these conditions are met.

\section{Linear Discontinuous Methods}

The LD methods which we now consider are derived from finite-element considerations. On the $j$ th spatial cell, let us define the cell-average flux $\psi_{m j}$ and cell-average "slope" $\hat{\psi}_{m j}$ by

$$
\begin{aligned}
& \psi_{m j}=\frac{1}{h_{j}} \int_{x_{j-1 / 2}}^{x_{j+1 / 2}} \psi_{m}(x) d x, \\
& \hat{\psi}_{m j}=\frac{6}{h_{j}^{2}} \int_{x_{j-1 / 2}}^{x_{j+1 / 2}}\left(x-x_{j}\right) \psi_{m}(x) d x .
\end{aligned}
$$

Then, if $\psi_{m}(x)$ is nearly a linear function of $x$ across the cell, we have

$$
\psi_{m}(x) \approx \psi_{m j}+\frac{2}{h_{j}}\left(x-x_{j}\right) \hat{\psi}_{m j}, \quad\left|x-x_{j}\right| \leqslant h_{j} / 2
$$

Likewise, we shall define constants $Q_{j}$ and $\hat{Q}_{j}$ in terms of the known source $Q(x)$ :

$$
\begin{aligned}
& Q_{j}=\frac{1}{h_{j}} \int_{x_{j-1 / 2}}^{x_{j+1 / 2}} Q(x) d x, \\
& \hat{Q}_{j}=\frac{6}{h_{j}^{2}} \int_{x_{j-1 / 2}}^{x_{j+1 / 2}}\left(x-x_{j}\right) Q(x) d x,
\end{aligned}
$$

and we have (approximately)

$$
Q(x) \approx Q_{j}+\frac{2}{h_{j}}\left(x-x_{j}\right) \hat{Q}_{j}, \quad\left|x-x_{j}\right|<h_{j} / 2 .
$$


The LD discretizations of problem (1.1) are now given by:

$$
\begin{gathered}
\frac{\mu_{m}}{h_{j}}\left(\psi_{m, j+1 / 2}-\psi_{m, j-1 / 2}\right)+\frac{\sigma_{T}}{\varepsilon} \psi_{m j} \\
=\left(\frac{\sigma_{T}}{\varepsilon}-\varepsilon \sigma_{A j}\right) \frac{1}{2} \sum_{n=1}^{N} \psi_{n} w_{n}+\frac{\varepsilon Q_{j}}{2}, \\
\frac{\mu_{m}}{\theta h_{j}}\left(\psi_{m, j+1 / 2}+\psi_{m, j-1 / 2}-2 \psi_{m j}\right)+\frac{\sigma_{T}}{\varepsilon} \hat{\psi}_{m j} \\
=\left(\frac{\sigma_{T}}{\varepsilon}-\varepsilon \sigma_{A j}\right) \frac{1}{2} \sum_{n-1}^{N} \hat{\psi}_{n} w_{n}+\frac{\varepsilon \hat{Q}_{j}}{2}, \\
\psi_{m j} \pm \hat{\psi}_{m j}=\psi_{m, j \pm 1 / 2}, \quad \mu_{m} \gtrless 0 . \\
\psi_{m, 1 / 2}=f_{m}, \quad \mu_{m}>0, \\
\psi_{m, J+1 / 2}=g_{m}, \quad \mu_{m}<0 .
\end{gathered}
$$

Equation (4.3a) is obtained by integrating the discrete ordinates form of Eq. (1.1a) over the $j$ th cell. Equation (4.3b) is obtained by multiplying the discrete-ordinates form of Eq. (1.1a) by $x-x_{j}$, integrating over the $j$ th cell, and setting $\theta=\frac{1}{3}$. It turns out to be useful, however, to consider other values of $\theta$, so in our analysis we shall allow $\theta$ to be a free parameter [16]. Within the context of the discrete-ordinates approximation, Eqs. (4.3a) and (4.3b), with $\theta=\frac{1}{3}$, are exact. Equation (4.3c), which is not exact, is obtained by applying Eq. (4.1c) at $x=x_{j+1 / 2}$ for $\mu_{m}>0$, and at $x=x_{j-1 / 2}$ for $\mu_{m}<0$. Finally, Eqs. (4.3d) and (4.3e) are obtained directly from Eqs. (1.1b) and (1.1c).

The asymptotic analysis of problem (4.3) proceeds just as in Section II. Thus, we introduce the ansatz (2.3) for the cell average fluxes and slopes and the cell edge fluxes into Eqs. (4.3), equate the coefficients of $\varepsilon^{-1}, \varepsilon^{0}$, and $\varepsilon^{1}$, and solve the resulting system of equations.

The two $O\left(\varepsilon^{-1}\right)$ equations are

$$
\begin{aligned}
& \sigma_{T j}\left(\psi_{m j}^{(0)}-\frac{1}{2} \sum_{n=1}^{N} \psi_{n j}^{(0)} w_{n}\right)=0 \\
& \sigma_{T j}\left(\hat{\psi}_{m j}^{(0)}-\frac{1}{2} \sum_{n=1}^{N} \hat{\psi}_{n j}^{(0)} w_{n}\right)=0 .
\end{aligned}
$$

These equations have the isotropic solutions

$$
\begin{aligned}
& \psi_{m j}^{(0)}=\frac{1}{2} \phi_{j}^{(0)}, \\
& \hat{\psi}_{m j}^{(0)}=\frac{1}{2} \hat{\phi}_{j}^{(0)},
\end{aligned}
$$

where $\phi_{j}^{(0)}$ and $\hat{\phi}_{j}^{(0)}$ are, at this point, undetermined. 
The $O\left(\varepsilon^{0}\right)$ equations can be written

$$
\begin{gathered}
\sigma_{T j}\left(\psi_{m j}^{(1)}-\frac{1}{2} \sum_{n=1}^{N} \psi_{n j}^{(1)} w_{n}\right)=-\frac{\mu_{m}}{h_{j}}\left(\psi_{m, j+1 / 2}^{(0)}-\psi_{m, j-1 / 2}^{(0)}\right), \\
\sigma_{T j}\left(\hat{\psi}_{m j}^{(1)}-\frac{1}{2} \sum_{n=1}^{N} \hat{\psi}_{n j}^{(1)} w_{n}\right)=-\frac{\mu_{m}}{\theta h_{j}}\left(\psi_{m, j+1 / 2}^{(0)}+\psi_{m, j-1 / 2}^{(0)}-\phi_{j}^{(0)}\right), \\
\psi_{m, j+1 / 2}^{(0)}=\left\{\begin{array}{lll}
f_{m}, & j=0, & \mu_{m}>0 \\
\frac{1}{2}\left(\phi_{j}^{(0)}+\hat{\phi}_{j}^{(0)}\right), & 1 \leqslant j \leqslant J, & \mu_{m}>0, \\
g_{m}, & j=J, & \mu_{m}<0, \\
\frac{1}{2}\left(\phi_{j+1}^{(0)}-\hat{\phi}_{j+1}^{(0)}\right), & 0 \leqslant j \leqslant J-1, & \mu_{m}<0 .
\end{array}\right.
\end{gathered}
$$

Multiplying Eqs. (4.6a) and (4.6b) by $w_{m}$ and summing over $m$, we find that the left sides vanish, and the right sides yield the solvability conditions

$$
0=\sum_{m=1}^{N} \mu_{m} \psi_{m, j+1 / 2}^{(0)} w_{m}, \quad 0 \leqslant j \leqslant J,
$$

which must be satisfied for a solution of Eqs. (4.6) to exist. Introducing Eqs. (4.6c) into (4.7), we easily obtain

$$
\begin{aligned}
\phi_{1}^{(0)}-\hat{\phi}_{1}^{(0)} & =\frac{4}{\gamma} \sum_{\mu_{m}>0} \mu_{m} f_{m} w_{m}, \\
\phi_{j}^{(0)}+\hat{\phi}_{j}^{(0)} & =\phi_{j+1}^{(0)}-\hat{\phi}_{j+1}^{(0)}, \quad 1 \leqslant j \leqslant J-1, \\
\phi_{J}^{(0)}+\hat{\phi}_{J}^{(0)} & =\frac{4}{\gamma} \sum_{\mu_{m}<0}\left|\mu_{m}\right| g_{m} w_{m} .
\end{aligned}
$$

If we make the definitions

$$
\begin{aligned}
\phi_{1 / 2}^{(0)} & \equiv \phi_{1}^{(0)}-\hat{\phi}_{1}^{(0)}, \\
\phi_{j+1 / 2}^{(0)} & \equiv \phi_{j}^{(0)}+\hat{\phi}_{j}^{(0)}=\phi_{j+1}^{(0)}-\hat{\phi}_{j+1}^{(0)}, \quad 1 \leqslant j \leqslant J-1, \\
\phi_{J+1 / 2}^{(0)} & \equiv \phi_{J}^{(0)}+\hat{\phi}_{J}^{(0)},
\end{aligned}
$$

then Eqs. (4.8) imply

$$
\begin{aligned}
\phi_{1 / 2}^{(0)} & =\frac{4}{\gamma} \sum_{\mu_{m}>0} \mu_{m} f_{m} w_{m}, \\
\phi_{j}^{(0)} & =\frac{1}{2}\left(\phi_{j+1 / 2}^{(0)}+\phi_{j-1 / 2}^{(0)}\right), \quad 1 \leqslant j \leqslant J,
\end{aligned}
$$




$$
\begin{aligned}
\hat{\phi}_{j}^{(0)} & =\frac{1}{2}\left(\phi_{j+1 / 2}^{(0)}-\phi_{j-1 / 2}^{(0)}\right), \quad 1 \leqslant j \leqslant J, \\
\phi_{J+1 / 2}^{(0)} & =\frac{4}{\gamma} \sum_{\mu_{m}<0}\left|\mu_{m}\right| g_{m} w_{m},
\end{aligned}
$$

and Eqs. (4.6c) can be written

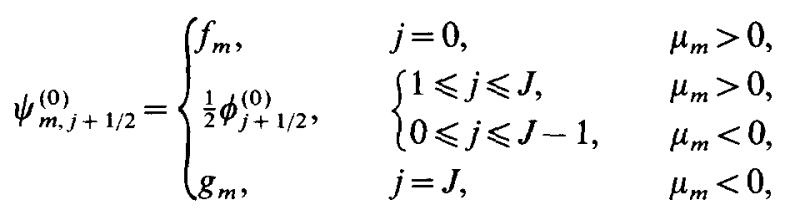

where $\phi_{1 / 2}^{(0)}$ and $\phi_{J+1 / 2}^{(0)}$ are given by Eqs. (4.10a) and (4.10d). The general solution of Eq. (4.6a) is now

$$
\psi_{m, j}^{(1)}=\frac{1}{2} \phi_{j}^{(1)}-\frac{\mu_{m}}{\sigma_{T j} h_{j}}\left(\psi_{m, j+1 / 2}^{(0)}-\psi_{m, j-1 / 2}^{(0)}\right), \quad 1 \leqslant j \leqslant J,
$$

where $\phi_{j}^{(1)}$ is, at this point, undetermined.

To determine the remaining $\phi_{j+1 / 2}^{(0)}$, we must consider the $O\left(\varepsilon^{1}\right)$ equations. The two of these which we will need are:

$$
\begin{aligned}
& \sigma_{T j}\left(\psi_{m j}^{(2)}-\frac{1}{2} \sum_{n=1}^{N} \psi_{n j}^{(2)} w_{n}\right) \\
& =-\frac{\mu_{m}}{h_{j}}\left(\psi_{m, j+1 / 2}^{(1)}-\psi_{m, j-1 / 2}^{(1)}\right)+\frac{1}{2}\left(-\sigma_{A j} \phi_{j}^{(0)}+Q_{j}\right), \\
& \sigma_{T j}\left(\hat{\psi}_{m j}^{(2)}-\frac{1}{2} \sum_{n=1}^{N} \hat{\psi}_{n j}^{(2)} w_{n}\right) \\
& =-\frac{\mu_{m}}{\theta h_{j}}\left(\psi_{m, j+1 / 2}^{(1)}+\psi_{m, j-1 / 2}^{(1)}-2 \psi_{m j}^{(1)}\right)+\frac{1}{2}\left(-\sigma_{A j} \hat{\phi}_{j}^{(0)}+\hat{Q}_{j}\right) .
\end{aligned}
$$

The solvability conditions for these equations are

$$
\begin{aligned}
& \vartheta_{j+1 / 2}^{(1)}-\vartheta_{j-1 / 2}^{(1)}=h_{j}\left(-\sigma_{A j} \phi_{j}^{(0)}+Q_{j}\right), \\
& \vartheta_{j+1 / 2}^{(1)}+\vartheta_{j-1 / 2}^{(1)}=2 \sum_{m=1}^{N} \mu_{m} \psi_{m j}^{(1)} w_{m}+\theta h_{j}\left(-\sigma_{A j} \hat{\phi}_{j}^{(0)}+\hat{Q}_{j}\right),
\end{aligned}
$$

where $\vartheta$ is defined by Eq. (2.26). Adding Eq. (4.14a) over the $j$ th and $(j+1)$ th cells, and subtracting Eq. (4.14b) over these same two cells, we obtain two expressions for $\vartheta_{j+3 / 2}^{(1)}-\vartheta_{j \sim 1 / 2}^{(1)}$. We equate these two expressions and get 


$$
\begin{aligned}
\sum_{m=1}^{N} \mu_{m}\left(\psi_{m, j+1}^{(1)}-\psi_{m j}^{(1)}\right) w_{m} \\
+\frac{1}{2}\left[\sigma_{A, j+1} h_{j+1}\left(\phi_{j+1}^{(0)}-\theta \hat{\phi}_{j+1}^{(0)}\right)+\sigma_{A j} h_{j}\left(\phi_{j}^{(0)}+\theta \hat{\phi}_{j}^{(0)}\right)\right] \\
=\frac{1}{2}\left[h_{j+1}\left(Q_{j+1}-\theta \hat{Q}_{j+1}\right)+h_{j}\left(Q_{j}+\theta \hat{Q}_{j}\right)\right], \quad 1 \leqslant j \leqslant J-1 .
\end{aligned}
$$

Now we combine this with Eqs. (4.10)-(4.12) and (3.22). To describe the results, let us first define $\Phi_{j+1 / 2}$ to be the solution of the diffusion problem

$$
\begin{gathered}
-\frac{1}{3 \sigma_{T, j+1} h_{j+1}}\left(\Phi_{j+3 / 2}-\Phi_{j+1 / 2}\right)+\frac{1}{3 \sigma_{T j} h_{j}}\left(\Phi_{j+1 / 2}-\Phi_{j-1 / 2}\right) \\
+\frac{1}{4}\left\{\sigma_{A, j+1} h_{j+1}\left[(1-\theta) \Phi_{j+3 / 2}+(1+\theta) \Phi_{j+1 / 2}\right]\right. \\
\left.+\sigma_{A j} h_{j}\left[(1+\theta) \Phi_{j+1 / 2}+(1-\theta) \Phi_{j-1 / 2}\right]\right\} \\
=\frac{1}{2}\left[h_{j+1}\left(Q_{j+1}-\theta \hat{Q}_{j+1}\right)+h_{j}\left(Q_{j}+\theta \hat{Q}_{j}\right)\right], \quad 1 \leqslant j \leqslant J-1, \\
\frac{1}{2} \Phi_{1 / 2}=\sum_{\mu_{n}>0}\left(\frac{3}{2} \mu_{n}^{2}+\frac{1}{\gamma} \mu_{n}\right) f_{n} w_{n}, \\
\frac{1}{2} \Phi_{J+1 / 2}=\sum_{\mu_{n}<0}\left(\frac{3}{2} \mu_{n}^{2}+\frac{1}{\gamma}\left|\mu_{n}\right|\right) g_{n} w_{n} .
\end{gathered}
$$

Then $\phi_{j+1 / 2}^{(0)}$ is defined in terms of $\Phi_{j+1 / 2}$ and Eqs. (4.10) by:

$$
\phi_{j+1 / 2}^{(0)}= \begin{cases}\frac{4}{\gamma} \sum_{\mu_{n}>0} \mu_{n} f_{n} w_{n}, & j=0, \\ \Phi_{j+1 / 2}, & 1 \leqslant j \leqslant J-1, \\ \frac{4}{\gamma} \sum_{\mu_{n}<0}\left|\mu_{n}\right| g_{n} w_{n}, & j=J .\end{cases}
$$

Finally, using Eqs. (4.5a), (4.10b), and (4.11), we obtain the following expressions for the cell-edge and cell-average angular fluxes:

$$
\psi_{m, j+1 / 2}=\left\{\begin{array}{lll}
f_{m}, & j=0, & \mu_{m}>0 \\
\frac{2}{\gamma} \sum_{\mu_{n}>0} \mu_{n} f_{n} w_{n}, & j=0, & \mu_{m}<0 \\
\frac{1}{2} \Phi_{j+1 / 2}, & 1 \leqslant j \leqslant J-1 \\
\frac{2}{\gamma} \sum_{\mu_{n}<0}\left|\mu_{n}\right| g_{n} w_{n}, & j=J, & \mu_{m}>0 \\
g_{m}, & j=J, & \mu_{m}<0
\end{array}\right\}+O(\varepsilon),
$$




$$
\psi_{m j}=\left\{\begin{array}{ll}
\frac{1}{4} \Phi_{3 / 2}+\frac{1}{\gamma} \sum_{\mu_{n}>0} \mu_{n} f_{n} w_{n}, & j=1 \\
\frac{1}{4}\left(\Phi_{j+1 / 2}+\Phi_{j-1 / 2}\right), & 2 \leqslant j \leqslant J-1 \\
\frac{1}{4} \Phi_{J-1 / 2}+\frac{1}{\gamma} \sum_{\mu_{n}<0}\left|\mu_{n}\right| g_{n} w_{n}, & j=J
\end{array}\right\}+O(\varepsilon) .
$$

Therefore, for all but the cells adjoining the two boundaries, the cell-edge and cell-average fluxes are completely determined by the problem (4.16). Equation (4.16a) is a stable and consistently differenced version of the diffusion equation (1.3a), and Eqs. (4.16b) and (4.16c) are very accurate representations of the diffusion boundary conditions (1.3b) and (1.3c) (see Eqs. (1.5)). In fact, if we consider a high-order quadrature set, for which $\gamma \approx 1$, then the weight function $\hat{W}(\mu)$ in Eqs. (4.16b) and $(4.16 \mathrm{c})$,

$$
\hat{W}(\mu)=\frac{3}{2} \mu^{2}+\mu
$$

satisfies

$$
\sup _{0 \leqslant \mu \leqslant 1}|W(\mu)-\hat{W}(\mu)|=0.018
$$

Therefore, the numerical solution in all but the two outermost cells adjoining the boundaries is determined by a stable and consistent discretization of the correct diffusion equation, and even though no effort has been made to resolve the boundary layers, the numerical diffusion boundary conditions very closely agree with the analytic diffusion boundary conditions.

The parameter $\theta$ appears in Eq. (4.16a), but not in (4.16b) or (4.16c). Although the standard value, $\theta=\frac{1}{3}$, arises as a natural consequence of the finite element procedure, the choice $\theta=1$ appears to be better for optically thick cells because it causes the absorption terms to collapse to a one-point rather than a three-point representation, making Eq. (4.16) more robust. We recommended the choice $\theta=1$ for optically thick cells.

We now turn our attention to the two cells adjoining the outer boundaries of the system. In these cells, the outermost cell-edge fluxes $\left[\psi_{m, 1 / 2}\right.$ and $\left.\psi_{m, J+1 / 2}\right]$ are, to leading order, unaffected by the diffusion problem (4.16), and the cell average fluxes $\left[\psi_{m, 1}\right.$ and $\left.\psi_{m, J}\right]$ are partly affected by this problem. We can estimate $\psi_{m, 1}$ and $\psi_{m, J}$ by arguing that since

$$
\begin{gathered}
\Phi_{3 / 2}=\Phi_{1 / 2}+O(\sigma h), \\
\Phi_{J-1 / 2}=\Phi_{J+1 / 2}+O(\sigma h),
\end{gathered}
$$

then, by Eqs. (4.16) and (4.19), 


$$
\begin{aligned}
& \psi_{m 1}=\sum_{\mu_{n}>0}\left(\frac{3}{4} \mu_{n}^{2}+\frac{3}{2 \gamma} \mu_{n}\right) f_{n} w_{n}+O(\sigma h)+O(\varepsilon), \\
& \psi_{m J}=\sum_{\mu_{n}<0}\left(\frac{3}{4} \mu_{n}^{2}+\frac{3}{2 \gamma}\left|\mu_{n}\right|\right) g_{n} w_{n}+O(\sigma h)+O(\varepsilon) .
\end{aligned}
$$

The weight function in these formulas, for high-order quadrature sets,

$$
\bar{W}(\mu)=\frac{3}{4} \mu^{2}+\frac{3}{2} \mu
$$

satisfies

$$
\sup _{0 \leqslant \mu \leqslant 1}|W(\mu)-\bar{W}(\mu)|=0.268
$$

Therefore, the cell-average fluxes in the two outermost "boundary" cells can have significantly larger errors than the corresponding fluxes in the "interior" cells. The LD solutions behave in a quantitatively different manner in the boundary cells because of the unresolved boundary layers in these cells at $x=0$ and $L$. It is quite surprising that the LD solutions arc so accurate in the interior cells, because these "see" information from the boundaries only as it is passed through the two (relatively inaccurate) boundary cells. Nevertheless, the numerical results that we have observed are in complete agreement with our theory.

\section{ACCURACY ON SySTEM BOUNDARIES}

In the previous sections we have determined the asymptotic limit of discretized versions of the transport problem (1.1) and have shown that, to varying degrees of accuracy, one obtains discretized versions of the diffusion problem (1.2) in the interior of the system. In this section we compare the analytical and numerical transport results as $\varepsilon \rightarrow 0$ on the outer boundaries of the system, at the points $x=0$ and $L$, where the diffusion solution (1.3) is not valid.

As $\varepsilon \rightarrow 0$, the analytic solution of problem (1.1) satisfies [9-11], at $x=0$, and $L$,

$$
\begin{aligned}
& \frac{1}{2} \int_{-1}^{1} \psi(0, \mu) d \mu=\int_{0}^{1} Z(\mu) f(\mu) d \mu, \\
& \frac{1}{2} \int_{-1}^{1} \psi(L, \mu) d \mu=\int_{-1}^{0} Z(-\mu) g(\mu) d \mu,
\end{aligned}
$$

where

$$
Z(\mu)=\frac{1}{X(-\mu)}\left[\int_{0}^{1} \frac{1}{X(-s)} d s\right]^{-1} .
$$

Equations (5.1a) and (5.1b) result from the fact that, to leading order, $\psi(0, \mu)$ for 
$\mu<0$ is the flux exiting a $c=1$ right half space $(x \geqslant 0)$ with incident flux $f(\mu)$, and $\psi(L, \mu)$ for $\mu>0$ is the flux exiting a $c=1$ left half space $(x \leqslant L)$ with incident flux $g(\mu)$. The function $Z(\mu)$ is smooth and well-approximated by the polynomial

$$
\tilde{Z}(\mu)=0.5174+0.954 \mu
$$

where

$$
\sup _{0 \leqslant \mu \leqslant 1}|Z(\mu)-\tilde{Z}(\mu)|=0.017
$$

For the DD method, we obtain from Eqs. (2.14), (2.20), and (2.21), that the angular fluxes exiting the system are highly anisotropic (i.e., distorted), and

$$
\begin{aligned}
& \frac{1}{2} \sum_{m=1}^{N} \psi_{m, 1 / 2} w_{m} \\
& \quad=\sum_{\mu_{m}>0}\left(\frac{1}{2}+\frac{\mu_{m}}{\gamma}\right) f_{m} w_{m}+(-1)^{J} \sum_{\mu_{m}<0}\left(\frac{1}{2}-\frac{\left|\mu_{m}\right|}{\gamma}\right) g_{m} w_{m}+O(\varepsilon), \\
& \frac{1}{2} \sum_{m=1}^{N} \psi_{m, J+1 / 2} w_{m} \\
& \quad=\sum_{\mu_{m}<0}\left(\frac{1}{2}+\frac{\left|\mu_{m}\right|}{\gamma}\right) g_{m} w_{m}+(-1)^{J} \sum_{\mu_{m}>0}\left(\frac{1}{2}-\frac{\mu_{m}}{\gamma}\right) f_{m} w_{m}+O(\varepsilon) .
\end{aligned}
$$

For the LD method, we obtain from Eq. (4.18) that the angular fluxes exiting the system are isotropic, and

$$
\begin{aligned}
\frac{1}{2} \sum_{m=1}^{N} \psi_{m, 1 / 2} w_{m} & =\sum_{\mu_{m}>0}\left(\frac{1}{2}+\frac{\mu_{m}}{\gamma}\right) f_{m} w_{m}+O(\varepsilon), \\
\frac{1}{2} \sum_{m=1}^{N} \psi_{m, J+1 / 2} w_{m} & =\sum_{\mu_{m}<0}\left(\frac{1}{2}+\frac{\left|\mu_{m}\right|}{\gamma}\right) g_{m} w_{m}+O(\varepsilon) .
\end{aligned}
$$

For a higher-order quadrature set, $\gamma \approx 1$, and the weight function $\hat{Z}(\mu)$ in Eqs. (5.5),

$$
\hat{Z}(\mu)=\frac{1}{2}+\mu \text {, }
$$

is a good approximation to $Z(\mu)$, satisfying

$$
\sup _{0 \leqslant \mu \leqslant 1}|Z(\mu)-\hat{Z}(\mu)|=0.045 \text {. }
$$

Therefore, the DD angular fluxes exiting the slab are highly distorted, the corresponding scalar fluxes at the system boundaries can be very inaccurate, and both generally will vary, depending on whether the system contains an even or odd number of cells. In contrast, the LD exiting angular fluxes are smooth, and the corresponding scalar fluxes at the system boundaries are quite accurate. 


\section{Numerical Results}

In this section we shall consider two slab-geometry transport problems that illustrate the theory developed above. The first problem is defined by:

$$
\begin{array}{rlrl}
\mu \frac{\partial}{\partial x} \psi(x, \mu)+\sigma_{T}(x) \psi(x, \mu) & =\frac{\sigma_{T}(x)}{2} \int_{-1}^{1} \psi\left(x, \mu^{\prime}\right) d \mu^{\prime}, & 0<x<11, \\
\psi(0, \mu) & =1, & & u>0, \\
\psi(11, \mu) & =0, & \mu>0, \\
\sigma_{T}(x) & = \begin{cases}2, & 0<x<1 \\
100, & 1<x<11,\end{cases} \\
\sigma_{S}(x) & = \begin{cases}0, & 0<x<1 \\
100, & 1<x<11 .\end{cases}
\end{array}
$$

The dimension of $x$ is $\mathrm{cm}$ and of $\sigma_{T}$ and $\sigma_{S}$ is $\mathrm{cm}^{-1}$. The system in this problem thus consists of a two mean-free path purely absorbing slab adjoining a one thousand mean-free path purely scattering slab. An isotropic incident angular flux $\psi(x, \mu)=1$ on the outer edge of the absorber attenuates to the anisotropic angular flux $\psi(x, \mu)=e^{-2 / \mu}$ that enters the thick, scattering region. We solved this problem using the $S_{16}$ Gauss-Legendre quadrature set and

$$
h= \begin{cases}0.1, & 0<x<1, \\ 10 / N, & 1<x<11,\end{cases}
$$

with various values of $N$. For $N$ not too large, the scattering region $1<x<11$ has

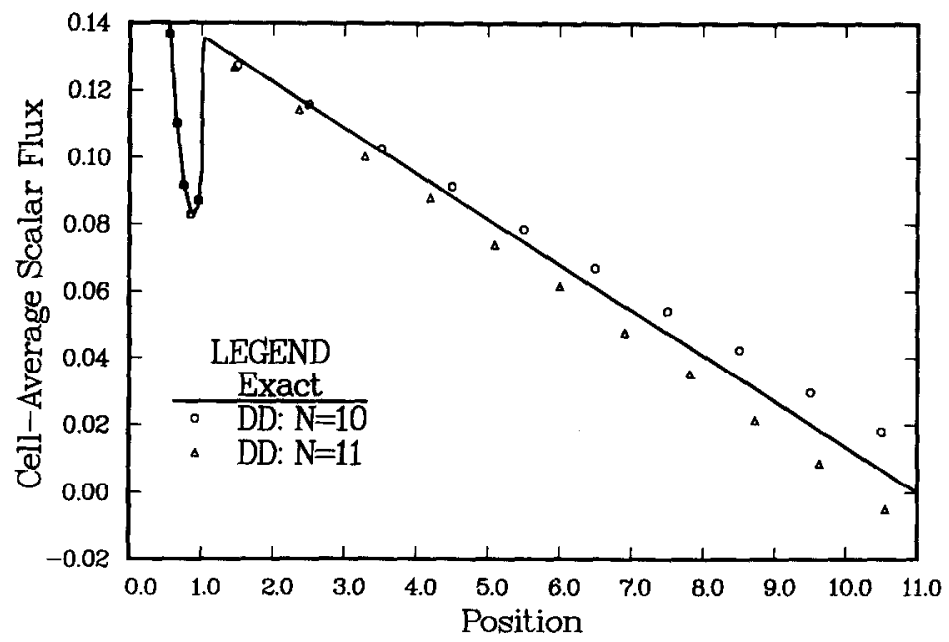

FIG. 1. Problem 1: Exact and DD cell-average scalar fluxes. 


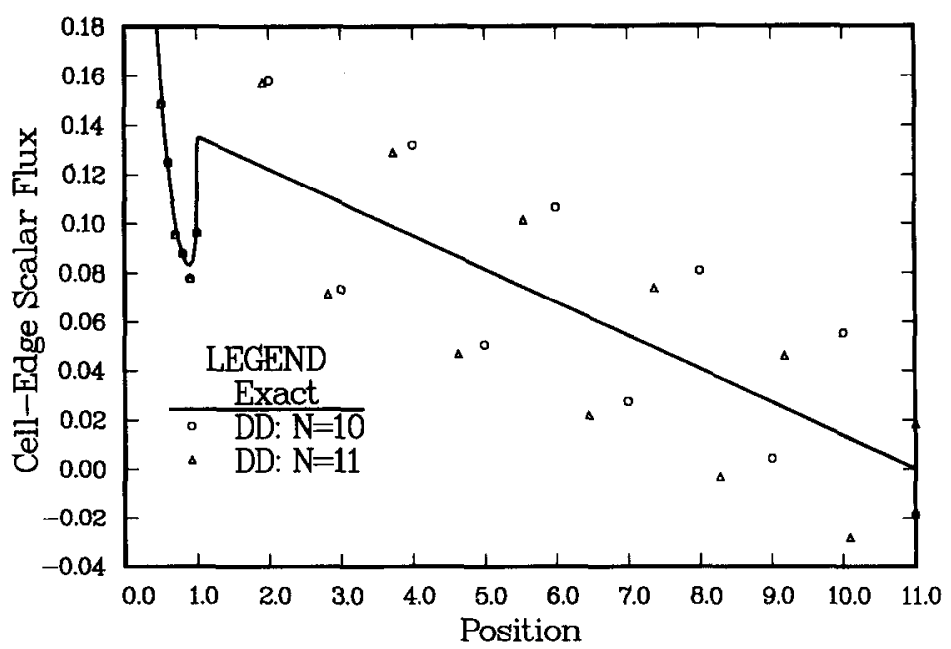

Fig. 2. Problem 1: Exact and DD cell-edge scalar fluxes.

cells that are optically thick, and the boundary layer at $x=1$ is not resolved. Away from the right and left boundaries of the scattering region, the exact angular flux is nearly isotropic and the spatial variation is nearly linear.

The "exact" (obtained using a very fine spatial mesh) scalar flux and the DD cell-average scalar fluxes for this problem are shown in Fig. 1, for $N=10$ and 11 . As predicted by our theory, the $N=10$ and 11 fluxes vary linearly across the scattering region, and both solutions agree near the boundary layer at $x=1$, but they increasingly disagree away from the boundary layer. The results for other even

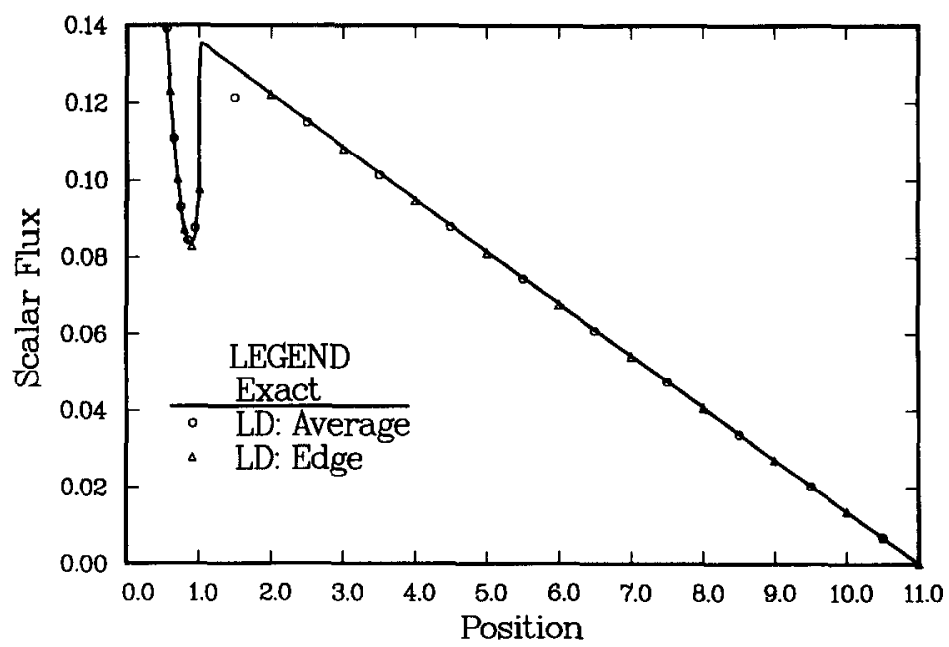

Fig. 3. Problem 1: Exact and LD scalar fluxes. 
values of $N$ lie nearly on the line drawn through the $N=10$ points in Fig. 1, and results for other odd values of $N$ lie nearly on the line drawn through the $N=11$ points in Fig. 1.

The exact scalar flux and the DD cell-edge scalar fluxes for this problem are plotted in Fig. 2, again for $N=10$ and 11. As predicted by our theory, these fluxes oscillate by substantial amounts around the exact solution.

The exact scalar flux and the LD cell edge and cell-average scalar fluxes for problem one are plotted in Fig. 3, for $N=10$. Other values of $N$ produce essentially the same results. As predicted by our theory, the LD solution is very accurate, except in the first cell (containing the unresolved boundary layer) in the purely scattering region.

Problem two is defined by

$$
\begin{aligned}
& \mu \frac{\partial}{\partial x} \psi(x, \mu)+\sigma_{T}(x) \psi(x, \mu)=\frac{\sigma_{T}(x)}{2} \int_{-1}^{1} \psi\left(x, \mu^{\prime}\right) d \mu^{\prime}+\frac{Q(x)}{2}, \quad 0<x<20, \\
& \psi(0, \mu)=0, \quad \mu>0, \\
& \psi(20, \mu)=0, \quad \mu<0, \\
& \sigma_{T}(x)=100, \\
& \sigma_{S}(x)=\left\{\begin{array}{lr}
90, & 0<x<10 \\
100, & 10<x<20
\end{array}\right. \\
& Q(x)=\left\{\begin{array}{lr}
10, & 0<x<10 \\
0, & 10<x<20 .
\end{array}\right.
\end{aligned}
$$

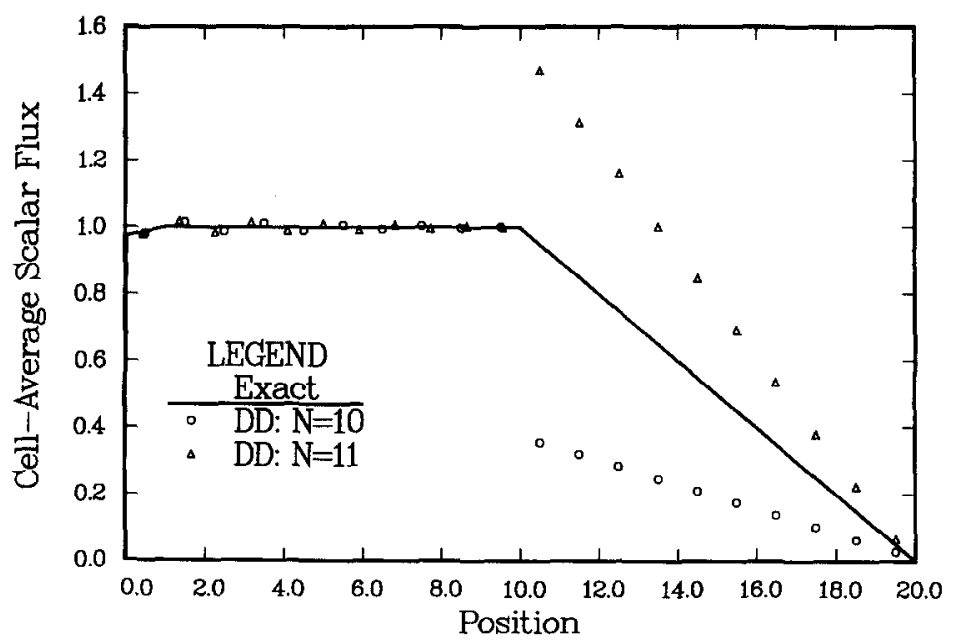

F)G. 4. Problem 2. Exact and DD cell-average scalar fluxes. 
The system in this problem consists of a 1000 mean free path slab, with absorption and a flat interior source, adjoining a 1000 mean free path purely scattering slab with no interior source. The exact solution of this problem is nearly constant, $\phi=1$, in the source region, and it varies nearly linearly from $\phi=1$ to $\phi=0$ as $x$ increases from 10 to 20. Although the source region is not "asymptotically diffusive" because $\sigma_{A}$ is not small, the solution of the standard diffusion approximation to the above problem is, nevertheless, highly accurate. We solved this problem using the $S_{8}$ quadrature set and

$$
h=\left\{\begin{array}{lr}
10 / N, & 1<x<10 \\
1.0, & 10<x<20
\end{array}\right.
$$

with various values of $N$.

The exact and DD cell-average scalar fluxes for this problem are plotted in Fig. 4, for $N=10$ and 11 . Although the DD fluxes are reasonably accurate in the source region, they are highly inaccurate in the scattering region. As before, results for other even values of $N$ lie nearly on the line drawn through the $N=10$ points in Fig. 4, and results for other odd values of $N$ lie nearly on the line drawn through the $N=11$ points in Fig. 4 .

The reason for the inaccuracies in these DD results can be seen in Fig. 5, which contains plots of the exact and the DD cell-edge scalar fluxes. Here we see that the DD cell-edge fluxes oscillate across the entire problem, and because the edge fluxes that leave the source region and enter the scattering region are the ones that "drive" the solution in the scattering region, large errors in these edge fluxes will generate comparably large errors in both the cell-edge and cell-average fluxes in the scattering region.

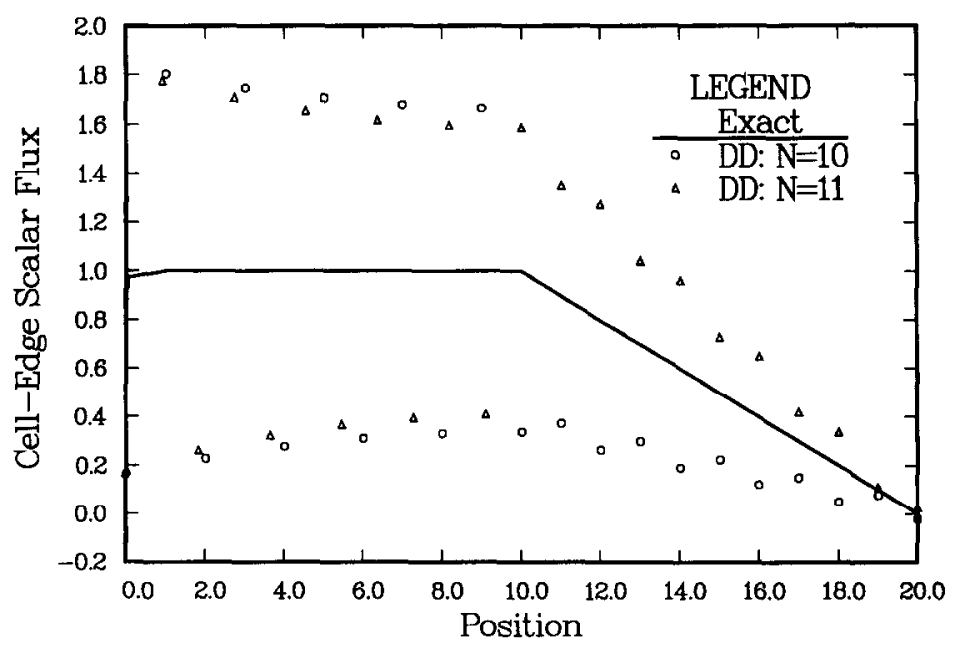

FIG. 5. Problem 2. Exact and DD cell-edge scalar fluxes. 


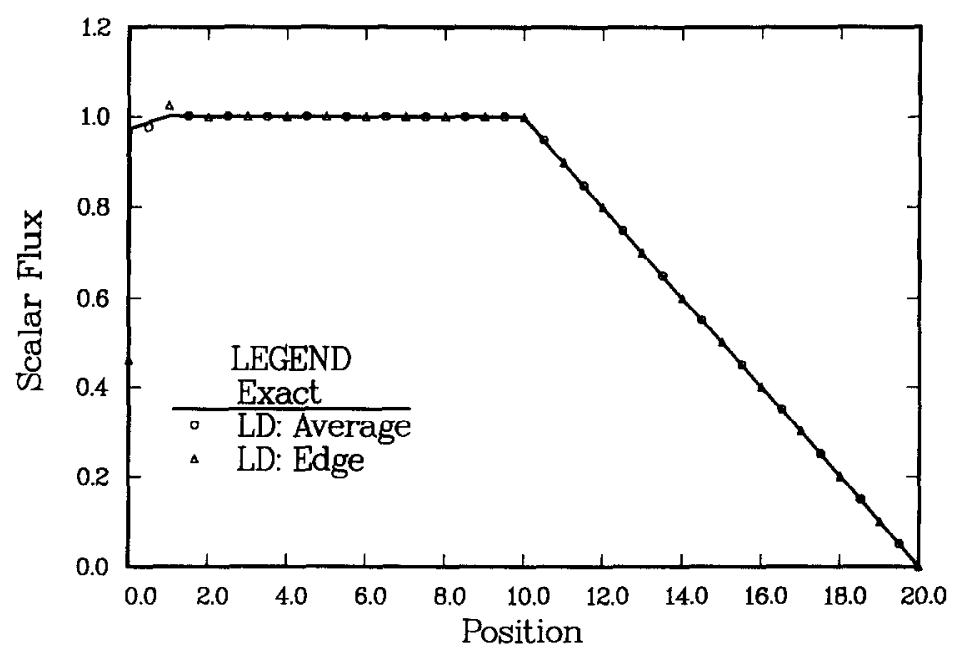

Fig. 6. Problem 2. Exact and LD scalar fluxes.

The exact and the LD cell-edge and cell-average scalar fluxes for this problem are plotted in Fig. 6, for $N=10$. Other values of $N$ produce essentially the same results. As in problem one, the LD solution is very accurate, except for the cell containing the boundary layer at $x=0$.

These two examples explicitly demonstrate that for diffusive transport problems containing optically thick spatial cells and unresolved boundary layers, the DD cell-edge and cell-average fluxes can have unacceptably large errors, while the LD cell-edge and cell-average fluxes have errors that are often negligible.

\section{Discussion}

In this article we have developed and tested an asymptotic method for determining the accuracy of numerical transport solutions for diffusive problems with optically thick spatial meshes and unresolved boundary layers. Our theory uses the fact that the analytic transport problem asymptotically tends to an analytic diffusion problem, by determining whether the discretized transport problem tends (in the same asymptotic limit) to the correct discretized diffusion problem.

Our results for the DD scheme are mixed; they show:

(a) if the prescribed incident fluxes satisfy Eqs. (2.32) (which happens, for example, if these fluxes are isotropic) then the cell-average fluxes will be accurate;

(b) if the prescribed incident fluxes are isotropic, then the cell-edge fluxes will be accurate; 
(c) for anisotropic incident boundary fluxes, the DD cell-edge and cell-average fluxes can be inaccurate, and these inaccuracies will depend on whether the number of cells in the system is even or odd.

Our results for the LD method are much more encouraging; the LD cell edge fluxes are very accurate everywhere, and cell average fluxes are very accurate on all cells except possibly those containing unresolved boundary layers. Moreover, the bounds on the LD errors which we determined (Eqs. (4.21), (4.26), and (5.6b)) are worst-case estimates that are rarely met in practice.

One of the serious objections to the LD method is that it requires more storage than the DD method-a factor of two more in one spatial dimension, of three in two spatial dimensions, and of four in three spatial dimensions. However, this LD storage penalty is compensated by the need to use a very fine spatial resolution of boundary layers with the DD method to achieve the accuracy that LD procedures with totally unresolved boundary layers.

Finally, we have examined another high-order differencing scheme based on a linear representation of $\psi$ in each cell-the linear moments method $[4,18]$, which is a 1-dimensional version of the linear-linear nodal method [19]; our asymptotic results are identical to those derived in Section IV for the LD method. It seems likely, therefore, that only marginal gains in accuracy can be achieved with other differencing schemes based on a linear representation of $\psi$ in a cell, and that to obtain significantly better results one will have to consider methods based on quadratic representations. This might be a fruitful avenue for further research.

\section{ACKNOWLEDGMENTS}

The authors thank Marvin Adams for some helpful discussions and a referee for some useful suggestions on an earlier version of this article. Much of the work of the first author (E.W.L.) was done during a visit at the Los Alamos National Laboratory. The work of the second author (J.E.M.) was performed under the auspices of the U.S. Department of Energy.

\section{REFERENCES}

1. E. W. Larsen, J. E. Morel, and W. F. Miller, JR., J. Comput. Phys. 69, 283 (1987).

2. E. E. LeWis AND W. F. Miller, JR., Computational Methods of Neutron Transport (Wiley Interscience, New York, 1984).

3. J. Pitkaranta and L. R. Scott, SiaM J. Numer. Anal. 20, 922 (1983).

4. E. W. Larsen and P. Nelson, SIAM J. Numer. Anal. 19, 334 (1982).

5. B. NetA AND H. D. Victory, JR., Numer. Funct. Anal. Optim. 5, 85 (1982).

6. C. Lecot, Transport Theory Statist. Phys. 15, 61 (1986).

7. J. E. Morel, Nucl. Sci. Eng. 79, 340 (1981).

8. R. E. Alcouffe, B. A. Clark, and E. W. Larsen, in Multiple Time Scales, edited by J. U. Brackbill and B. Cohen (Academic Press, Orlando, 1985), p. 73.

9. G. J. Habetler and B. J. Matkowsky, J. Math. Phys. 16, 846 (1975).

10. E. W. Larsen and J. B. Keller, J. Math. Phys. 15, 75 (1974). 
11. K. M. CASE AND P. F. Zweifel, Linear Transport Theory (Addison-Wesley, Reading, MA, 1967).

12. T. R. Hill, Los Alamos Scientific Laboratory Report No. LA-5990-MS, June 1975. (unpublished).

13. R. E. Alcouffe, E. W. Larsen, W. F. Miller, Jr., and B. R. Wienke, Nucl. Sci. Eng. 71,111 (1979).

14. J. E. Morel and T. R. Hill, "A New Class of $S_{N}$ Differencing Schemes," in Radiation Transport; October I, 1986-December 31, 1986, edited by R. D. O’Dell and H. Byers, Los Alamos National Laboratory Report No. LA-11154-PR, February, 1988 (unpublished).

15. E. W. LarSEN AND J. E. Morel, "Analysis of Several $S_{N}$ Differencing Schemes in the Thick Diffusion Limit," in Radiation Transport; April 1, 1987-June 30, 1987, edited by R. D. O'Dell and H. M. Byers, Los Alamos National Laboratory Report LA-11188-PR, April 1988 (unpublished).

16. C. D. LEVERMORE, private communication.

17. A. H. Stroud and D. SeCrest, Gaussian Quadrature Formulas (Prentice Hall, Englewood Cliffs, NJ, 1966).

18. R. Vaidyanathan, Nucl. Sci. Eng. 71, 46 (1979).

19. W. F. Walters AND R. D. O'Dell, Proc. Conf. Advances in Mathematical Methods for the Solution of Nuclear Engineering Problems, Vol. 1, p. 115 (Munich, April 27-29, 1980, American Nuclear Society, 1980). 